\title{
Dynamical evolution of dense star clusters in galactic nuclei
}

\author{
Jaroslav Haas and Ladislav Šubr \\ Charles University in Prague, Faculty of Mathematics and Physics, \\ V Holešovičkách 2, 18000, Prague, Czech Republic \\ email: haas@sirrah.troja.mff.cuni.cz, subr@sirrah.troja.mff.cuni.cz
}

\begin{abstract}
By means of direct numerical $N$-body modeling, we investigate the orbital evolution of an initially thin, central mass dominated stellar disk. We include the perturbative gravitational influence of an extended spherically symmetric star cluster and the mutual gravitational interaction of the stars within the disk. Our results show that the two-body relaxation of the disk leads to significant changes of its radial density profile. In particular, the disk naturally evolves, for a variety of initial configurations, a similar broken power-law surface density profile. Hence, it appears that the single power-law surface density profile $\propto R^{-2}$ suggested by various authors to describe the young stellar disk observed in the Sgr A* region does not match theoretical expectations.
\end{abstract}

Keywords. methods: n-body simulations, stellar dynamics, Galaxy: nucleus

\section{Model}

The components of the studied system are: (i) the dominating central mass, SMBH, represented by the Keplerian potential, (ii) the stellar disk treated as a group of gravitating particles on initially circular orbits, and (iii) the spherical cluster which we model either by a static analytical gravitational potential or by a larger number of gravitating particles. The dynamical evolution of the system is followed numerically, by means of the $N$-body integration code NBODY6 (Aarseth 2003).

\section{Surface density profile evolution}

Our calculations show that the evolution of the disk leads, for a wide set of its initial density profiles, to a broken power-law surface density profile: $\Sigma(R) \propto R^{\beta_{\mathrm{i}}}$. Furthermore, we find that its shape is not influenced by the possible presence of the spherical cluster in the system.

With respect to the young stellar disk observed in the Sgr A* region, our results are in a good agreement with the broken power-law reported by Buchholz et al. (2009) for this system $\left(\beta_{1}=-1.08, \beta_{2}=-3.46\right.$; see Figure 1$)$, in contrary to the single power-law $\Sigma(R) \propto R^{-2}$ suggested by various authors (e.g., Paumard et al. 2006, Lu et al. 2009).

\section{Eccentricity distribution evolution}

Unlike the surface density profile, the evolution of the eccentricity distribution in the disk is affected by the cluster gravity if the cluster is modeled by a group of gravitating particles. In such a case, the eccentricity evolution is slightly accelerated (solid line in Figure 2) in comparison to the situation with no cluster present (dashed line in Figure 2) or the cluster represented by an analytical potential (dotted line in Figure 2). 


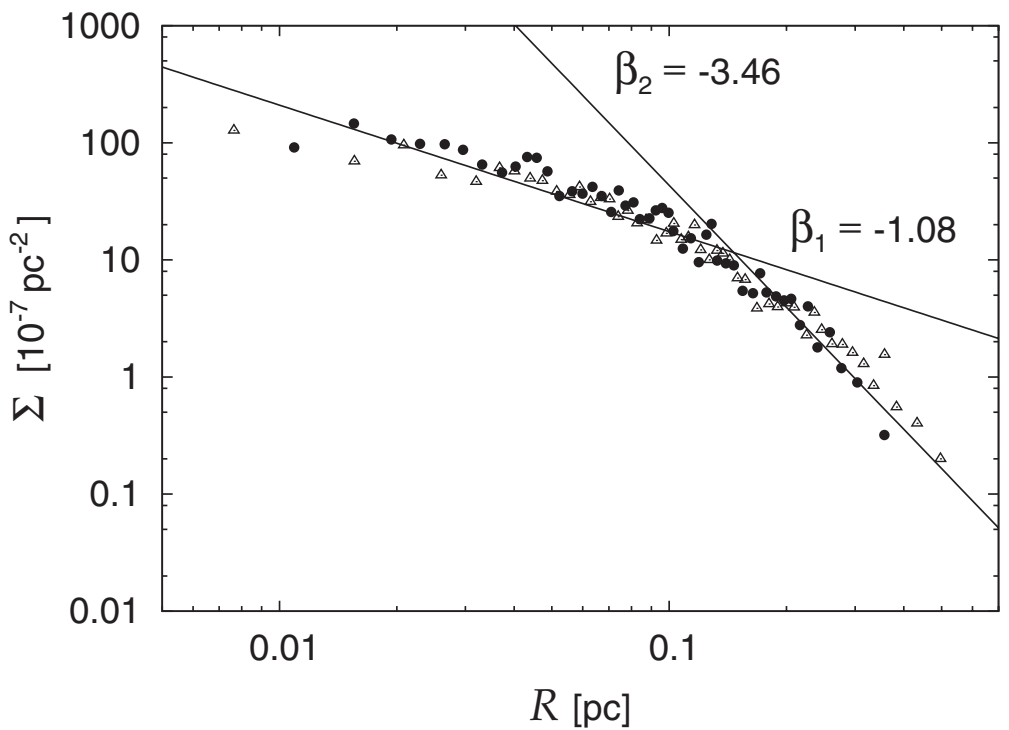

Figure 1. Projected surface density profile of the disk at $t=6 \mathrm{Myr}$ for two different initial configurations. Empty triangles: spherical cluster modeled as a large number of gravitating particles, initial distribution of the radii of the individual stellar orbits in the disk: $\mathrm{d} N / \mathrm{d} R \propto R^{-1}$. Filled circles: no spherical cluster present, initial distribution of the radii of the individual stellar orbits in the disk: $\mathrm{d} N / \mathrm{d} R \propto R^{-2}$.

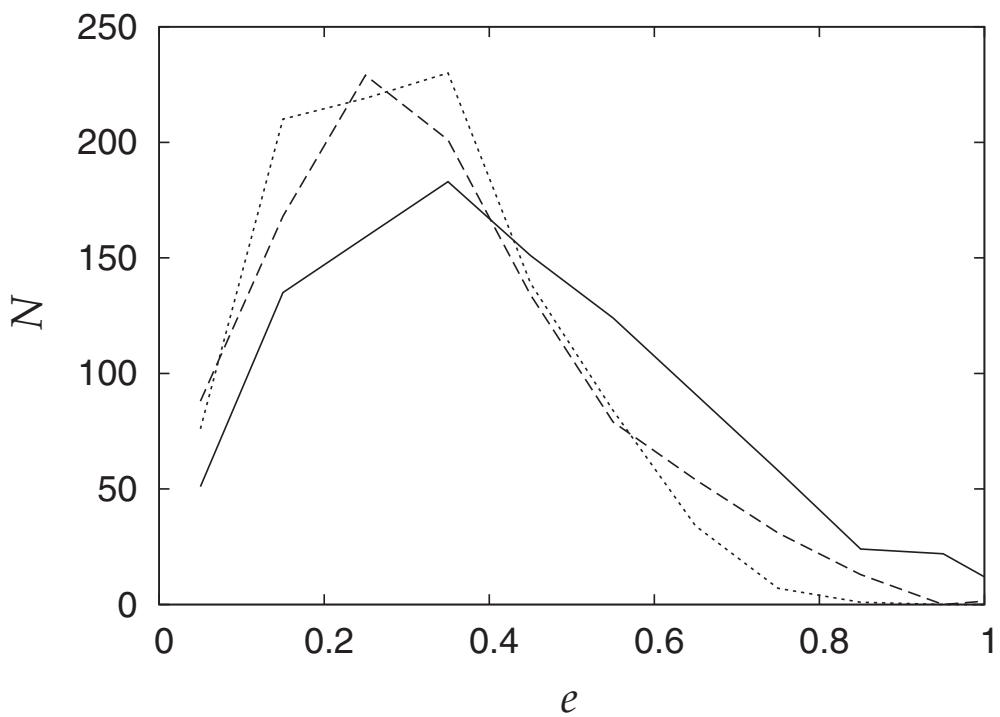

Figure 2. Eccentricity distribution in the disk at $t=6 \mathrm{Myr}$ for three different treatments of the cluster gravity: no cluster present (dashed), analytical potential (dotted) and a large number of gravitating particles (solid).

\section{Acknowledgements}

This work has been supported by project LD12065 of the research programme COST CZ of the Czech Ministry of Education, Youth and Sports. 


\section{References}

Aarseth, S. J. 2003, Gravitational N-Body Simulations (Cambridge: Cambridge Univ. Press) Buchholz, R. M., Schödel, R., \& Eckart, A. 2009, A\& A 499, 483

Lu, J. R., Ghez, A. M., Hornstein, S. D., Morris, M. R., Becklin, E. E., \& Matthews, K. 2009, ApJ 690, 1463

Paumard, T., Genzel, R., Martins, F., Nayakshin, S., Beloborodov, A. M., Levin, Y., Trippe, S., Eisenhauer, F., Ott, T., Gillessen, S., Abuter, R., Cuadra, J., Alexander, T., \& Sternberg, A. 2006, ApJ 643, 1011 